\title{
The Dimensionality
}

\author{
Sunil Sharma \\ Independent Researcher \\ Ulhasnagar, India
}

\begin{abstract}
This thesis is all about dimensions and the law of Visual, Physical and Time (V,P,T) dimension which succeed to discovered more than 9 dimension (with examples) which includes current three spatial dimensions as well. General idea of the law is to show that the Dimensions are categorized by phases not by sequences, and Visual, Physical, and Time are the phases the contains their own specific dimensions e.g. Initial Dimensions like Length, Mass and Infinite Time, respectively. Although this paper covers some of the major topics which are (i) Non-Existence of 4th dimension, (ii) Understanding the law of VPT Dimension (iii) Types (iv) Phases (v) Rules of Representation (vi) General term and Numerical term, (vii) Human Dimension, and some uses. This research can be used in various fields like physics, cosmology studies, mathematics, architecture, etc. which generates massive impact on science development. The topics which are not entertain in this paper are Dimensional formula, some theories claim higher dimensions and higher dimensions like 5th 6th and so on.
\end{abstract}

Keywords:- Phases, Law Of VPT Dimension, 4th Dimension, Dimensions of Phase (DP), Types, Rules, Human Dimension.

\section{INTRODUCTION}

There is no clearly defined definition for the word 'Dimension' in science till now because some definitions include direction [1],[2] whereas some excludes Mass and Temperature[1],[2],[3] which is the major issue for further dimensions errors. Directions like up, down, left, right, etc. are never be the part of Dimension but it is the part of length, breadth, and height and because of this, Mass and Temperature never be introduced or considered.

Every object that exist contain their own specific or unique dimension, precisely every human being has different value dimension as compared to each other even their length, breadth, and height matches. It is almost impossible to match the same value. For now, focusing on the definition of Dimension, 'It is the measurable extent of an object/s in terms of physical quantities such as Length, Breadth, Height, Mass, Temperature, Time.' According to the Law of Visual, Physical and Time (V,P,T) Dimension, it is categorized by phases not in sequences like current spatial dimension. Length as 1D, length + breadth as 2D and length + breadth theight as 3D are the three spatial dimensions were as Spacetime combines Time with 3-dimensional space fabric to fuse single 4th dimensional continuum [4]. Matter of fact, Mass and Temperature cannot be introduced in such way, but it is the important part of dimension that cannot be ignored simultaneously.

\section{A. Nonexistence of 4th Dimension}

Let us try to understand, how Mass and Temperature affects Space-time as $4^{\text {th }}$ dimension (4D). Assumed an Imaginary Apple in having length, breadth, and height along with one dimension of time [4] (assume certain amount of time.) in short 4D. If we try to touch Apple with hands, we will not be able to differentiate its existence because of two major reason (i) Yet mass is not the part of it, there will no force generates (ii) due to absence of temperature we unable to feel any differences. Therefore, the imaginary apple does not follow any laws and rules of physics as well as thermodynamics. This object will behave as a 'Holographic 3D project' [5] for certain time.

To ensure this imaginary 4D Apple turn into a realworld object, Mass and Temperature plays major role. As a result, $4^{\text {th }}$ dimension cannot be existing in sequence, as mass and temperature does not fit into it. Similarly, when we differentiate the dimensions of Living and Non-living object/s Time creates massive difference and it shows itself a different dimension (section II B 3). Hence spacetime could be a concept but it cannot be considered as $4^{\text {th }}$ dimension.

In this research, the Law of VPT Dimension aligns Mass, Temperature, Spatial Dimension and Time dimension by distributing them in suitable phases. Although it provides a set of rules which can be applied universally.

\section{UNDERSTANDING THE LAW OF VPT DIMENSION}

The law of VPT dimension states that Dimension are categorized by phases not by sequence which allows to assign specific Physical properties and Time dimension under a single system of dimension (Analyzing Dimension of object/s in a particular manner). The categorization of Dimension is known as Phase and it has their own interconnected dimensions which are known as Dimension of phase (DP). According to the law, there are 3 phase and 9 DP were found existed till now and plus one dimension is assumption as Time Travel (section II B 3 d). Visual, Physical and Time are the phase contain 3, 3 and 3+1 DP, respectively. There are high scopes for future discovering dimension which can be placed into suitable phase and position, respectively. For example, Tesseract [6] might take the place of visual's $4^{\text {th }}$ dimension if it proves that it has an extra axis. 
Every object has two fundamental rules of dimension

1) Dimension of phase (DP) can be occur naturally (independently) or Interdependent on another dimension of phase.

2) Not every object stays into one fixed dimension, it can be change or might be change or keeps changing continously into different (especially value dimension).

Here are some important topics that are covered in this section as follow:

A) Types of dimension

B) Phases and their DP

\section{A) Types of Dimension}

Additional, Subtractional and Initial are the three types of dimension which determines that the DP is formed by either adding or subtracting components or it is form by root dimension. Every new DP which is formed by previous dimension (DP) along with the addition of X-factor are the Additional type. (here $\mathrm{X}$-factor is a component which cause new dimension.) Example, the current $2^{\text {nd }}$ dimension i.e. V2 dimension contains $1^{\text {st }}$ dimension (V1) i.e. length along with the addition of breadth. Similarly, if the DP is formed by subtracting any existing component to extract new DP is Subtractional type. Only $3^{\text {rd }}$ dimension of physical phase shows Subtractional type. Those DP which are connected from root dimension or zeroth dimension are known as Initial type. Basically, it is the origin of further dimension and every first DP of each phase is Initial type.

\section{B) Phases and their DP}

This is a detailed study about the Phases and DPs, also shown in the fig.1.

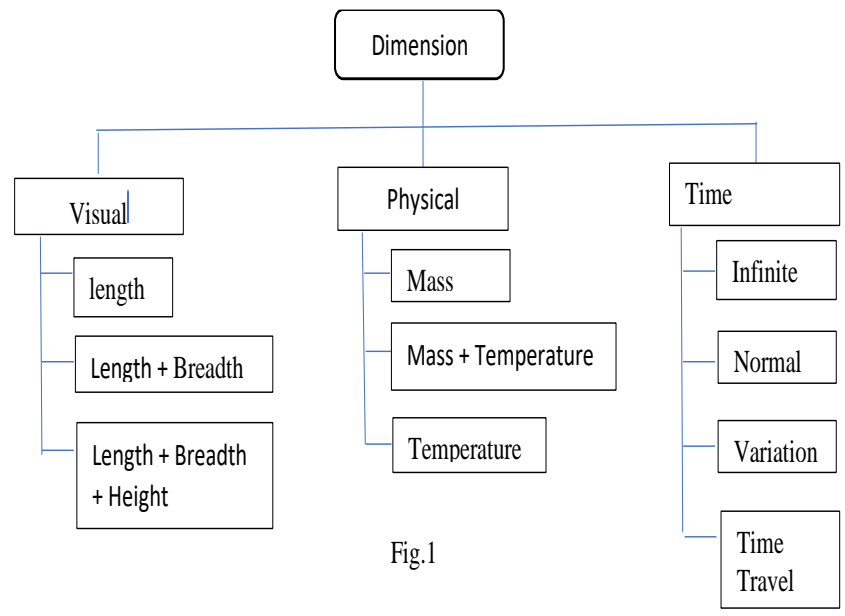

The study as follows:

1) Visual phase

2) Physical phase

3) Time phase

1) Visual phase:

It is the phase where the objects visual appearance or visibility is the factor of analyses like length, breath, and height. The Dimensions of this phase are the current three Spatial dimension which is formed by Length, Breadth and Height. i.e. Length, Length+ Breadth and Length+ Breadth
+ Height as first, second and third. The first dimension of visual phase is the initial type as it is formed by root or zeroth dimension and rest are the Addition type.

Visual phase is denoted by its initial capital letter ' $\mathrm{V}$ ' in general terms and the DP are represented as V1, V2 and V3, respectively.

\section{2) Physical phase:}

It is the phase where objects physical properties i.e. Mass and Temperature are the factor of analyses. This phase contains three DP as follow Mass, Mass+ Temperature, Temperature as first, second and third DP. Although it follows Initial, additional and Subtractional types, respectively. (Note: $3^{\text {rd }}$ dimensional of physical phase is the only Subtractional type as Mass is removed so we can extract new dimension i.e. Temperature.)

Physical phase is denoted by its initial capital letter ' $\mathrm{P}$ ' in the General term and its DP are represented as P1, P2 and P3 dimension, respectively.

\section{3) Time phase:}

It is the phase where the objects Time Length is the factor of measuring. The basic component of this phase is the flow of time. Although, this phase contains more than 3 DP where the possibility of time travel is counted as $4^{\text {th }} \mathrm{DP}$. Infinite time, Absolute time and Variation time are first, second and third dimension of time phase, respectively. Infinite time is an initial type whereas Absolute time and Variation time are the additional type of dimension.

Time phase is denoted by its initial capital letter ' $T$ ' in general term.

Let us understand these DP in detail as follows:

a) Infinite Time

b) Absolute Time

c) Variation Time

d) Time Travel (assumed Dimension)

a) Infinite Time: It is the DP where time exist in Infinite form such as it starts from infinity and ends at infinity which also provide immortality. For example, the smallest constitute of matter i.e. Atom [7] and the universe shows the same property. The origin of the atom and the universe is still mystery, but it might be started from infinity that is why the question beyond big bang [8] is always be the active point. Just like 2D object cannot look 3D object, similarly the object which is not in this DP can never find its origin. 'This could be the relation between the quantum to universe level.'

The representation of this DP is denoted by ' $\mathrm{T} 1$ ' in General term and ' $\infty$ ' is the value in Numerical term.

b) Normal Time: It is the DP where time exist in Absolute form that means it has a proper start and end points with constant flow of time. This dimension is created Infinite Time along with the addition of time period. Basically, it is $\mathrm{X}$-axis forward flow of time with constant velocity. 
Almost every non-living thing are the example of this dimension. Sometimes the object in this DP shows the property of infinite time. Almost every non-living object are the example of this dimension. This DP is denoted by $\mathrm{T} 2$ dimension in general term.

c) Variation Time: It is the DP where time exist in Variate form or relative time which means it has birth and death as time period but with inconstant flow if time. For example, A person on earth and on mars have some difference in their time flow even they both same origins i.e. both object's point of start. In short, they both suffers different flow of time in respect to another object. This dimension has two axes forward flowing time where $\mathrm{x}$ axis represent time length shows variation in time flow. This DP is denoted by T3 dimension in general term.

d) Time Travel: This DP is an assumption dimension where time flow of an object might change its direction in backward and forward. Here, time exist in a completely different form such as $\mathrm{X}$-Traveling state where $\mathrm{x}$ is the additional factor cause extra dimension. There are no such examples are found yet, but still there is possibility of it in future.

\section{RULES OF REPRESENTATION}

This set of rules to represent the dimension of any object is divided into two terms

a) General term

b) Numerical term.

General term is for the group of objects mean dimension where it is categorized it basic dimensional form whereas Numerical term is for the specific detailed value of the dimension of an object. The rules are as follows:

a) Rules for General term:

1. Every phase is represented by its capital initial letter.

Ex. Physical phase is written as ' $\mathrm{P}$ '.

2. The sequel number of dimensions i.e. DP is mentioned after the phase.

Ex. Length, breadth and height is written as 'V3'.

3. Sequence of phases must be Visual, Physical and Time, respectively.

4. The separation between the phases is done by using the symbol of semicolon (;) and Dimension at the end.

Ex. V3;P2;T3 Dimension.

5. If the phase is absent or not mentioned, then it is denoted by 0 th dimension

Ex. In the case of holographic image of kangaroo contain $x-$ $\mathrm{y}-\mathrm{z}$ axis but physical phase is absent so it will be represented by V3;P0;T2 Dimension.

b) Rules for Numerical term:

1. The Numerical term is written below the General term and it should be in square bracket.

2. The value of the DP is written in the curved bracket and its value should be separated by using the symbol of comma (,).
3. Semicolon (;) is used to separate the phases

4. If the value of DP is absent, then the numerical value will be denoted by $\mathrm{x}$.

5. If the value of phase is the $0^{\text {th }}$ dimension or absent, then its numerical term will be excluded.

\section{CONCLUSION AND RESULT}

1. This research finds 9 proper dimensions and plus one for time travel (assumed).

2. Successfully align mass, temperature, time and 3 spatial dimensions.

3. Provides a set of rules which can be applied universally.

4. From this research we can analyze the dimension of planets, living and non-living things and every object that exist in the universe.

5. Showing the similarity between the universe and quantum level by infinite time dimension.

6. By using the Law of VPT Dimensions, we can analyze the proper dimension of Human/s, its General term and Numerical term.

Example. If a person has $159 \mathrm{~cm}$ of height, $41 \mathrm{~cm}$ of length and $30 \mathrm{~cm}$ of width, the weight of the person is 75 kilograms and having average body temperature. The persons age is $25 y e a r s$ and 4 months. Then the dimension of the person is represented as follow. General term: $\mathrm{V} 3 ; \mathrm{P} 2 ; \mathrm{T} 3 \mathrm{Dimension}$
Numerical term: $[(41 \mathrm{~cm}, 30 \mathrm{~cm}, 159 \mathrm{~cm}) ;(75 \mathrm{~kg}$,
$\left.37^{\circ} \mathrm{C}\right) ;(25$ years and $\left.4 \mathrm{months})\right]$

7. This law can be used in various fields such as

i. In physics, study can discover new future dimensions, also to extract the proper dimensions of object/s.

ii. In cosmology, we can use this to analyze the dimension of planets, stars, exoplanets, etc.

iii. In Mathematics, we can calculate the numerical term dimension of object/s.

iv. In Architecture, numerical term can be used for representing the structures and models.

v. In Biology, we can use this to represent the dimension of humans

vi. Some other uses in daily basis, etc.

Future active area. precisely.

This research may help to study Blackhole [9] more

It may be helps to understand how time travel might be possible.

Helps to find future discovering dimension which can be placed according to its suitable place.

Can be useful to analyze the dimension of new elements, matter, objects, etc. 


\section{REFERENCES}

[1]. Cambridge University Press. (August 2020). Definition of Dimension. https://dictionary.cambridge.org/dictionary/english/dim ension

[2]. Oxford Learners Dictionaries. (N.D). Definition of dimension https://www.oxfordlearnersdictionaries.com/definition/a merican_english/dimension

[3]. Collins Dictionaries. (N.D) Definition of Dimension https://www.collinsdictionary.com/dictionary/english/di mension

[4]. Encyclopædia Britannica, inc. (05 october 2018) Spacetime https://www.britannica.com/science/space-time

[5]. Magic Holo. (N.D) what is a 3D Hologram https://magic-holo.com/en/what-is-a-3d-hologram/

[6]. LeiosOS (19 November 2016) Understanding 4D - The Tesseract LeiosOS

[7]. Encyclopædia Britannica, inc. (04 Feb.2020), Atom https://www.britannica.com/science/atom

[8]. Dr. Don Lincoln (1 april 2020) What happened before the big bang. Fermilab

[9]. NASA (22 AUG 2018), What Is a Black Hole? https://www.nasa.gov/audience/forstudents/k-

4/stories/nasa-knows/what-is-a-black-hole-k4.html 